\title{
Urinary Steroid Values as a Guide to Prognosis in Breast Cancer
}

\author{
K. A. AHLQUIST,* M.SC., M.C.B., F.R.I.C. ; A. W. JACKSON,* M.D., F.F.R. \\ J. G. STEWART,* M.A., M.B., CH.B., F.F.R.
}

Brit. med. F., 1968, 1, 217-221

Bulbrook, Greenwood, and Hayward (1960) published data relating urinary steroid excretion to response after ablative endocrine surgery (hypophysectomy or adrenalectomy) in advanced breast cancer. The impetus for this study derived from their experience that no clinical method was known which could select patients likely to respond to such treatment. The excretions of 17-hydroxycorticosteroids (17-OHCS) and of 11-deoxy-17-oxosteroids (11-DOOS) were particularly investigated, and of the latter group aetiocholanolone ( $3 \alpha$-hydroxy$5 \beta$-androstan-17-one) was found to be of most significance. On the basis of these studies statistical considerations led to the presentation of an expression-80-80 (17-OHCS mg./24 $\mathrm{hr}$.) + aetiocholanolone $(\mu \mathrm{g} . / 24 \mathrm{hr}$.) - which on appropriate substitution of found values gave rise to the "discriminant function." The discriminant was frequently positive in subjects responsive to such ablative procedures and negative in subjects showing no response.

Later publications from the same group of workers suggested that the discriminant appeared to be of some prognostic value in early disease. A large proportion of patients with early disease were found to have negative discriminants, this being very uncommon in normal subjects (Bulbrook, Hayward, Spicer, and Thomas, 1962). Furthermore, that group of patients with a positive discriminant at the time of mastectomy was found to have a lower recurrence rate and higher survival rate than the group with a negative discriminant (Bulbrook, Hayward, and Thomas, 1964). These various developments have given rise to a continuing series of publications from the same centre, reviewed by Atkins (1966). Recent publications in a similar context from other centres have been restricted to considerations of selection for endocrine ablation (Wilson, Crocker, Fairgrieve, Bartholomay, Emerson, and Moore, 1967 ; Juret, Hayem, and Flaisler, 1964 ; Kumaoka, Abe, Sakauchi, Takatani, and Kusama, 1966).

The foundation of such developments has been empirical. The in vivo biosynthesis of dehydroepiandrosterone, $\Delta^{4}$ androsten-3,17-dione, and cortisol by the human adrenal gland has been studied in patients with advanced breast cancer in whom this organ was infused in situ with precursor $\Delta^{5}-$ pregnenolone (tritiated) (Deshpande, Jenson, Bulbrook, and Doouss, 1967). The authors conclude, "there is a highly significant correlation between the biosynthesis of these compounds and the urinary excretion of 11-deoxy-17-oxosteroids and 17-hydroxycorticosteroids as represented by a urinary discriminant function." The nature of the interrelation of the latter factors with prognosis in breast cancer still remains a matter for conjecture. Nevertheless, if such investigations result in more rational and effective treatment of individual patients then their use is fully justified.

We have investigated early and late cases of breast cancer treated in this hospital by established procedures of surgery, radiotherapy, and hormone therapy. Facilities for the determination of aetiocholanolone were not at first available. It is, however, reasonable to suppose that aetiocholanolone excretion is closely related to the total excretion of 11-DOOS. Accordingly we earlier measured the latter and recorded the

- Christie Hospital and Holt Radium Institute, Manchester 20. ratio of (total) 11-DOOS to $17-\mathrm{OHCS}$ for our subjects. At a later stage it was found practicable to estimate aetiocholanolone by a gas-chromatographic method, and the discriminant could then be calculated. We present data relating these two values, and comment on their application to our patients.

Work concurrently carried out at another centre by Miller, Durant, Jacobs, and Allison (1967), using methods somewhat different from ours, introduced the ratio of 11-DOOS to 17-OHCS as an "alternative discriminant." In a study applied retrospectively to their own series of cases, Thomas, Bulbrook, and Hayward (1967) have shown such a ratio to give the same correlation with response to endocrine ablation as did their discriminant.

\section{Methods}

Urinary 17-OHCS were measured by a modification of the method of Few (1961). For the final extraction ethylene dichloride was used. Results were expressed in terms of dehydroepiandrosterone. The methods used to measure the urinary excretions of total 11-DOOS and of aetiocholanolone were closely modelled on those of Bulbrook's laboratory (Thomas and Bulbrook, 1966). All Zimmerman colorimetry differed from the practice in the latter in that organic base was used in place of potassium hydroxide (James and De Jong, 1961; Ahlquist, 1967). We believe that the consequent differences in chromogenicity would lead to no more than a minor discrepancy. Towards the end of the clinical work a cross-check was made with Bulbrook's laboratory, the results showing a good measure of agreement (unpublished data). In brief, urine was successively subjected to solvent extraction, glucuronidase hydrolysis, solvolysis, and paper chromatography. Divided aliquots of eluted 11-DOOS were estimated by $(a)$ the Zimmerman reaction (for total 11-DOOS) and (b) gas chromatography (for aetiocholanolone: proportionality calculation).

We have examined urine from 37 normal women, 63 cases of early breast cancer, and 107 cases of late breast cancer. The normal subjects were paramedical workers and their relatives and friends. Urine samples (24-hour) were taken either 10 days after mastectomy for patients having surgery in this hospital or immediately on admission here for postoperative radiotherapy, and always before $x$-ray artificial menopause. The late cases were initially sampled before hormone treatment or at least two weeks free of any such treatment. Observations on subsequent serial values are also presented in both early and late cases. Urine was collected without preservative, returned to the laboratory promptly, and an aliquot stored at $-18^{\circ}$ C. for brief periods, if necessary, before analysis. Stale urines were rejected.

Early cases are defined as those in which treatment was given with curative intent (stages 1, 2, or 3-Manchester staging). These cases were given such surgical treatment as was thought appropriate, with local radiotherapy and $x$-ray artificial menopause added according to Manchester principles (Paterson and Russell, 1959a).

Late cases are defined as those which by virtue of extent of local recurrence or metastatic disease were thought to be 
beyond radical treatment by surgery or radiotherapy. Such patients were treated according to the following policy:

(a) Premenopausal patients and those up to two years postmenopausal received $x$-ray artificial menopause with the addition of androgen therapy if no response was obtained.

(b) Patients two to five years' postmenopausal received androgens.

(c) Patients more than five years' postmenopausal received stilboestrol.

(d) A failure to respond or a relapse after the above therapy was generally followed by high-dose prednisone, and ablative operation (yttrium implant of the pituitary or adrenalectomy) was considered thereafter.

(e) A small number of patients were treated at some stage with cyclophosphamide.

Throughout this regimen symptoms have dictated treatment. In 5 of the 107 patients under observation hormone therapy has not yet become necessary. A breakdown of initial and subsequent treatments is given in Table $\mathrm{I}$.

TABLR I.-Treatment Given in 102 Cases of Late Breast Cancer

\begin{tabular}{|c|c|c|c|c|c|c|}
\hline \multicolumn{4}{|c|}{ Type of Therapy } & $\underset{\text { Treatment }}{\text { Initial }}$ & $\begin{array}{l}\text { Subsequent } \\
\text { Treatments }\end{array}$ & $\begin{array}{l}\text { No. } \\
\text { Responding }\end{array}$ \\
\hline $\begin{array}{l}\text { X-ray artificial men } \\
\text { Androgens } \\
\text { Stilboestrol } \\
\text { Prednisone }\end{array}$ & $\begin{array}{c}\text { ause } \\
\cdots \\
\cdots \\
\cdots \\
\cdots \\
\cdots\end{array}$ & $\begin{array}{l}\ddot{y} \\
\cdots \\
\cdots \\
\cdots\end{array}$ & \begin{tabular}{l|l}
$\cdots$ & \\
$\cdots$ & \\
$\cdots$ & \\
$\cdots$ & \\
$\cdots$ &
\end{tabular} & $\begin{array}{r}14 \\
35 \\
50 \\
2 \\
1 \\
-\end{array}$ & $\begin{array}{r}\overline{14} \\
5 \\
35 \\
6 \\
3 \\
7\end{array}$ & $\begin{array}{r}2 \\
5 \\
11 \\
5 \\
2 \\
3\end{array}$ \\
\hline
\end{tabular}

We have not attempted to correlate response to ablative operations with preoperative values of the ratio, since at that time most of our patients are taking high-dose prednisone, rendering the estimation invalid. However, we believe that the value of the discriminant has already been demonstrated in this context (see Bulbrook, 1965).

\section{Results}

\section{Relation Between Ratio and Discriminant}

The age distribution of the subjects is shown in Fig. 1. It will be seen that the main part lies below the age of 65 years;

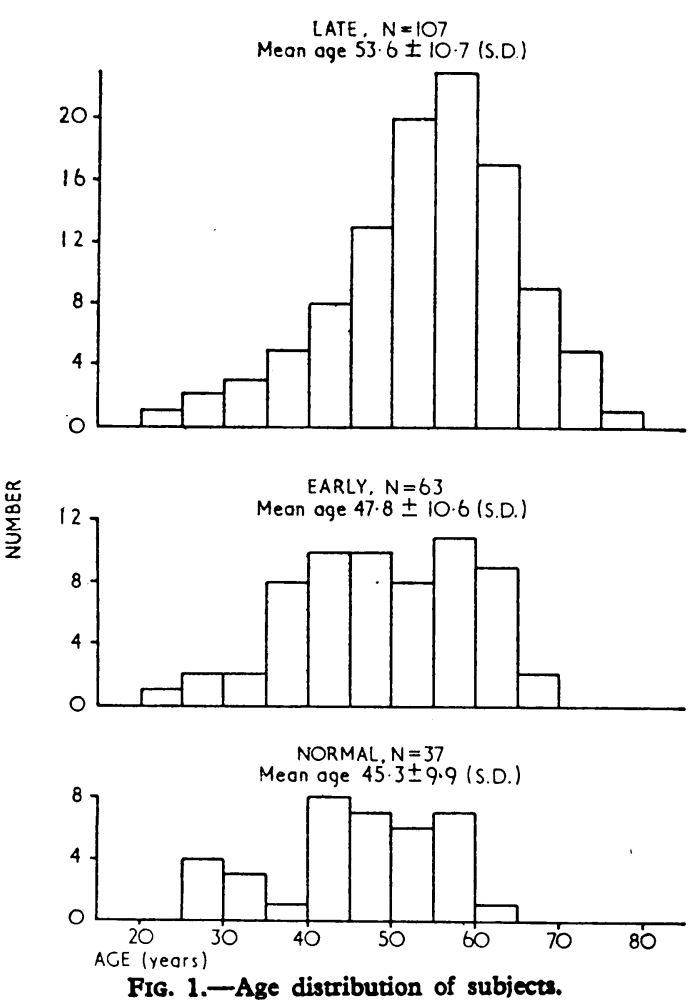

only 15/107 late cases and 2/63 early cases exceed this age. The early and normal groups are similar, but the late cases are somewhat older. It was found on statistical analysis that the ratio falls with increasing age, for both normal and breast cancer subjects (normal, $\mathrm{r}=-0.61, \mathrm{P}<0.001$; breast cancer, $r=-0.41, P<0.001$ ), as also reported by Miller et al. (1967).

In Figs. 2 and 3 are shown related values of the ratio and the discriminant for urine samples from 37 normal women and from 72 patients with breast cancer (both early and late) in whom both investigations were carried out. The regression lines are as indicated, the $\mathrm{x}$ intercepts (ratio) being 0.20 for the normal group and 0.15 for the cancer group. This difference in intercept could in part be determined by the age bias already commented on. It is seen that, with two exceptions only, the ratio was found to exceed 0.20 in the normal group ; no negative discriminants were found. In the cancer group ratio

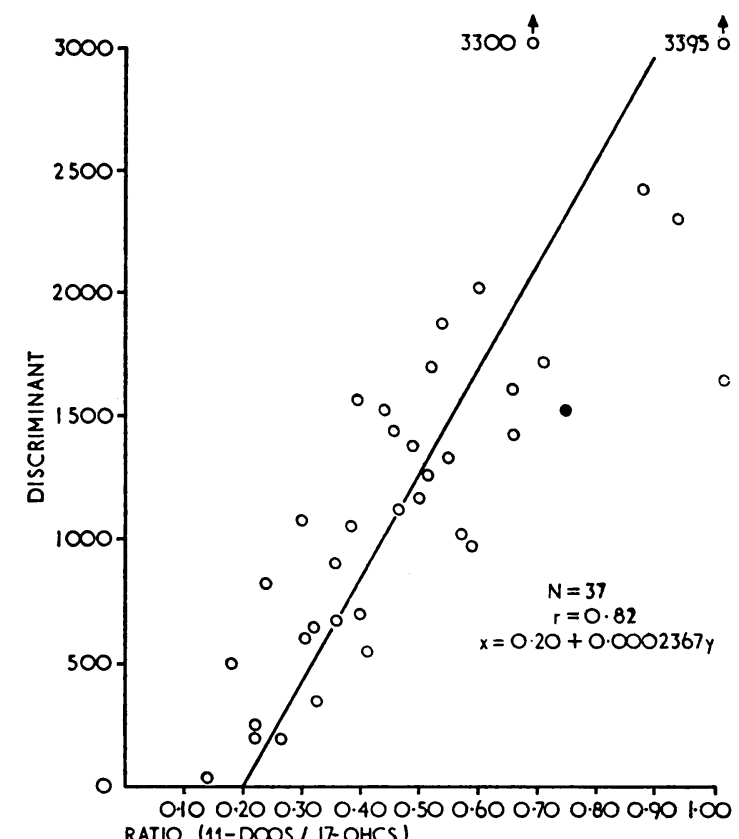

Fig 2.- Related values of the ratio and discriminant found in 37 normal women.

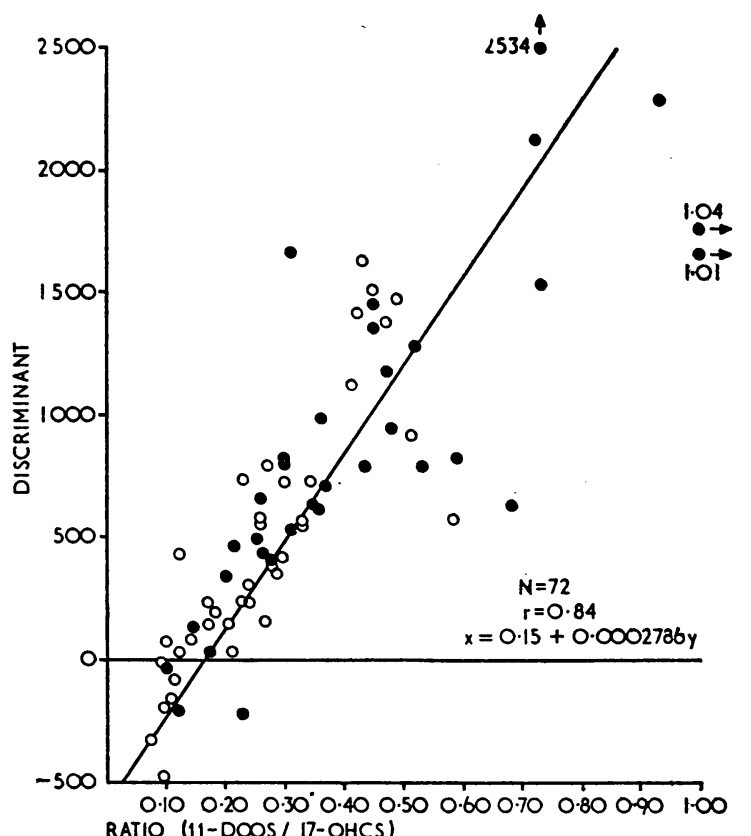

Fig. 3.-Related values of the ratio and discriminant found in 72 cases of breast cancer. Early cases; $O$ Late cases. 
values below 0.15 may be expected to correlate with a negative discriminant. Of 13 values less than 0.15 recorded, however, only eight were found to relate with a negative discriminant. Of the total nine negative discriminants recorded eight had ratio values less than 0.15 . On the basis of Fig. 3 we may thus classify ratio values below 0.15 as "negative discriminant" in a statistical sense. The probability of a particular ratio relating to a negative discriminant becomes much higher as the ratio falls : below 0.10 we may expect a negative discriminant with greater confidence.

\section{Early Cases}

The ratio values found with the 63 early cases are plotted in Fig. 4 according to stage and in terms of the observed subsequent progress of the disease. Clinical assessment was made at periods ranging from six months to two and a half years after the urine analysis. In those cases in which recurrence or metastasis occurred the general pattern appears random, and no helpful guidance regarding individual prognosis has yet been observed. The recurrence pattern at 12 months has also been examined. In this preliminary analysis the mean ratio of patients whose disease has recurred or metastasized is similar to that of patients remaining well at 12 months. One patient (stage 1) with a persistently high ratio (initially 0.85 ) developed a possible second primary in
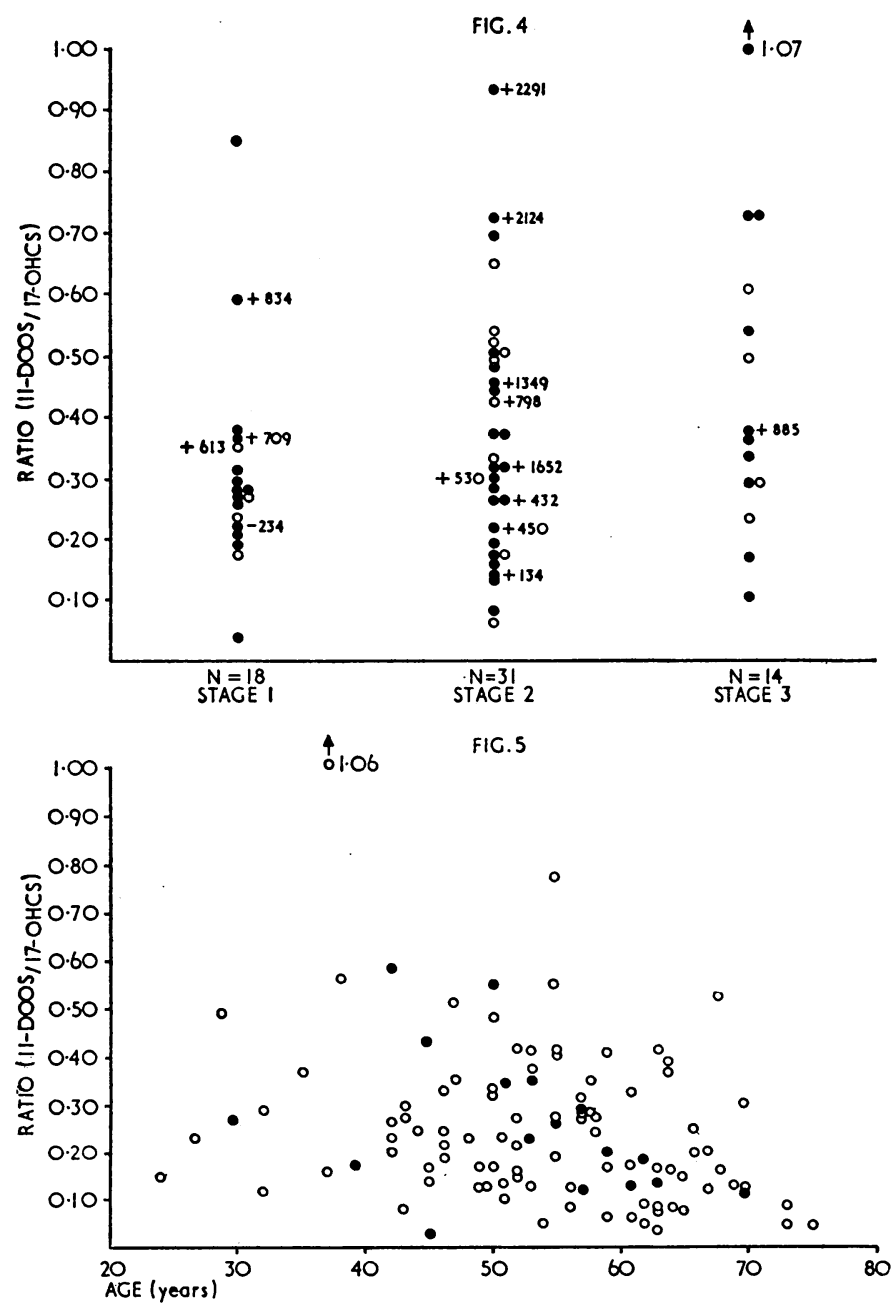

FIG. 4.-Values for the ratio found for 63 cases of early breast cancer. The associated values of the discriminant, where known, are also given. Clinical assessment was made at periods ranging from six months to two and a half years after the urine analysis. Fitients remaining well; Patients with recurrence or metastasis. FIg. 5.-Values of the ratio
found for 107 cases of late breast cancer. Patients responding to treatment; $O$ Patients failing to respond. the contralateral breast at nine months after the first radical mastectomy. She remained well after a further local mastectomy and has been classed in Fig. 4 as a well case.

\section{Late Cases}

Response to therapy has been assessed as follows: (1) unequivocal objective response-all known disease showing measurable regression; and (2) less marked objective response-regression but quantitatively less definite.

The duration of such response has ranged from 3 to 30 months, with a mean of 10 months, but some cases are still in remission. Subjective improvement alone, so common after prednisone and on occasion after androgens, has not been rated as response.

In Fig. 5 is given a scatter diagram of values of the ratio found in 107 cases of late breast cancer. Of 102 patients having had hormone therapy, 17 exhibiting an unequivocal objective response have been given a distinguishing symbol. If this diagram is viewed in relation to an arbitrary datum line of 0.15 it is seen that 12 responders had a ratio above the line, 5 below. A further 10 cases showed a less marked objective response. If these are added to the group of unequivocal responders the pattern in relation to this datum line becomes: for responders, 20 above and 7 below; for non-responders, 57 above and 23 below.

The relation between response rate and ratio has also been examined for particular treatment groups. Neither here nor when considering the overall management of these patients has a correlation between response and ratio been established.

\section{Serial Values}

Early Cases.-In 21 early cases repeat analyses were carried out ; the ratio values for this group are shown in Table II. It has been shown that prophylactic $x$-ray artificial menopause can improve prognosis in early breast cancer (Paterson and

TABLE II.-Serial values of the Ratio (11-DOOS/17-OHCS) in 21 Early

\begin{tabular}{|c|c|c|c|c|c|c|}
\hline \multicolumn{7}{|c|}{ Cases } \\
\hline 1 & 2* & 3* & $4^{*}$ & $5^{*}$ & $6^{*}$ & $7 *$ \\
\hline 0.85 & 0.37 & 0.69 & 1.07 & 0.17 & & 0.10 \\
\hline $1.04(8)$ & $0.52(15)$ & $0.20(17)$ & $1.22(9)$ & $0.30(6)$ & $0.27(2)$ & $0.11(20)$ \\
\hline & $\begin{array}{l}0.51(22) \\
+1,286\end{array}$ & & $\begin{array}{l}1.01(16) \\
+1,659\end{array}$ & & & \\
\hline $8^{*}$ & 9* & $10^{*}$ & $11^{*}$ & $12^{*}$ & $13^{*}$ & $14^{*}$ \\
\hline 0.33 & 0.59 & 0.60 & 0.31 & 0.22 & 0.14 & 0.45 \\
\hline $\begin{array}{l}0.30(10) \\
+819\end{array}$ & $\begin{array}{l}0.53(5) \\
+794 \\
0.71(8) \\
+815\end{array}$ & $\begin{array}{l}0.47(6) \\
+1,183\end{array}$ & $\begin{array}{l}0.36(15) \\
+9993 \\
0.34(17) \\
+635\end{array}$ & $\begin{array}{l}0.68(2) \\
+645 \\
0.73(4) \\
+2,534\end{array}$ & $\begin{array}{l}0.10(7) \\
-38\end{array}$ & $\begin{array}{r}0.45(6) \\
+1,450\end{array}$ \\
\hline $15^{*}$ & $16^{*}$ & $17^{*}$ & $18 *$ & 19 & 20 & $21^{*}$ \\
\hline 0.29 & 0.37 & 0.49 & 0.30 & 0.23 & 0.49 & 0.43 \\
\hline $\begin{array}{l}0.73(12) \\
+1,533\end{array}$ & $\begin{array}{l}0.26(7) \\
+659\end{array}$ & $\begin{array}{l}0.48(11) \\
+935\end{array}$ & $\begin{array}{l}0.25(4) \\
+493\end{array}$ & $0.28(10)$ & $0.08(10)$ & $\begin{array}{l}+798 \\
0.20(9) \\
+90\end{array}$ \\
\hline
\end{tabular}

Serial values of the ratio obtained for 21 early cases are displayed vertically (asterisk denotes prophylactic $x$-ray artificial menopause). The initial value is followed by later values, with the time elapsed (months) shown in parentheses.

Russell, 1959b). In 18 patients the ratio was determined before $x$-ray artificial menopause and this determination was repeated some months later. It is apparent that in most cases the ratio remained at a fairly steady level. Cases 12 and 15 showed a marked increase for no evident reason. Case 7 remained well at 20 months, though with a low ratio and negative discriminant. In three cases (Nos. 3, 20, and 21) a marked fall in the ratio was noted; the first of these was well at the repeat analysis, the last two had recurred. 
Late Cases.-In 34 late cases serial values of the ratio were recorded over periods extending from 1 to 27 months from the initial assay. In most cases the patient was off all hormones at the time of urine sampling for the later values but a few patients were taking stilboestrol. The levels showed considerably more variation than in the early cases. Several cases showed a fall in the ratio as the disease progressed. A fall in 11-DOOS accompanied by a rise in 17-OHCS, producing a decrease in the ratio, is known to be a general feature of terminal illness. However, instances were found where a low ratio was maintained in long-term survival. One patient showed a remarkable rise in ratio as her general condition improved after cyclophosphamide therapy. Values found in illustrative cases are shown in Fig. 6.

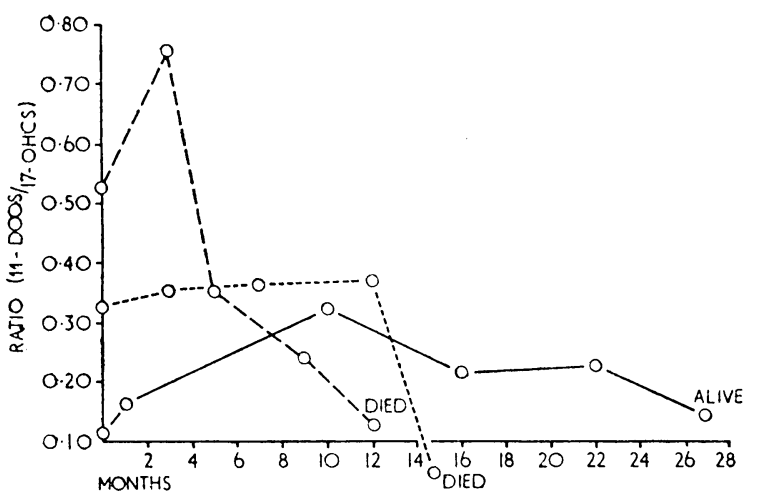

FIG. 6.-Serial values of the ratio found in late breast cancer-illustrative cases.

Initial hopes that response of individual patients to hormone therapy or $x$-ray artificial menopause would be associated with a consistent change in serial values did not materialize.

\section{Discussion}

The relation we have explored between the ratio and the discriminant reveals a pattern similar to that noted earlier by Miller et al. (1967) in introducing their "alternative discriminant" (11-DOOS/17-OHCS). However, only two cases in which aetiocholanolone was less than $30 \%$ of the total 11-DOOS were encountered in our study.

The original application of the discriminant is reported to be of continuing value for the optimal selection of cases for ablative surgery (Bulbrook, 1965). Earlier reports, however, indicating an appreciable proportion of negative discriminants in early breast cancer (Bulbrook et al., 1962, 1964) and which prompted our interest, are not supported by the present study. Our own findings in the early group may be summarized: ratio $6 / 63<0.15$; negative discriminant $1 / 12$. They thus indicate a very small proportion of cases with a presumptively negative discriminant. It is, however, proper to observe that it is possible that our calculated discriminant may deviate slightly in certain cases from the value which would be found in Bulbrook's laboratory, for reasons made apparent in the Methods section.

It has been reported that "the amounts of steroids found in the urine (10 days after mastectomy) are not necessarily characteristic of the amounts found at other times in the course of the disease" (Bulbrook and Hayward, 1966). Our own serial studies on 21 patients show no consistent difference over the periods examined.

The limitations of the established methods of analysis demanded for the investigation of hormonal status in cancer patients have been reviewed by Bulbrook (1966). In embarking on our study it was an initial stipulation that subjects should be "off all drugs." It became apparent that such a rule is generally impossible to maintain under practical circumstances. Several ill patients cannot be denied pain relief, nor is full control over self-medication practicable in outpatients. Even the ubiquitous anodyne aspirin may be a source of error; it is well known that salicylate metabolites may compete for glucuronidase (Stempfel, Sidbury, and Migeon, 1960). In the course of our investigations it became the practice to screen all urines with ferric chloride ; salicylatepositive urines were then processed with a double amount of glucuronidase. In about half a dozen instances, including two "normal" subjects, the methods employed gave indeterminate values. These cases have been excluded. It is entirely feasible that concealed errors may be quite common. It must be accepted that a proportion of results may be spurious, and it should be emphasized that individual results should be interpreted with caution.

It has been remarked that "it is unlikely that the discriminant will be of prognostic use in old patients" (Bulbrook et al., 1964). In this hospital $46 \%$ of patients treated for breast cancer are $\geqslant 60$ years of age and $33 \%$ are $\geqslant 65$. We have attempted to exclude patients over 65, and thus have been obliged to disqualify a large proportion of our patients from consideration.

The investigations reported in this article have led us to conclude that study of the ratio or of the discriminant has given us no useful prognostic indication for individual patients. We are not in a position to comment on the original application of the discriminant-that is, selection for endocrine ablative surgery-for reasons given earlier. In this

TABLE III.-Yttrium Ablation of Pituitary Related to Previous Responses to Hormone Therapy (142 Cases)

\begin{tabular}{|c|c|c|c|c|c|c|}
\hline \multirow{2}{*}{\multicolumn{4}{|c|}{ Hormone Response }} & \multirow{3}{*}{$\begin{array}{c}\text { No. of Cases } \\
73 \\
69\end{array}$} & \multicolumn{2}{|c|}{ Response to Yttrium Ablation } \\
\hline & & & & & No. & $\%$ \\
\hline $\begin{array}{l}\text { Negative } \\
\text { Positive... }\end{array}$ & $\because$ & $\because$ & 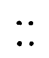 & & $\begin{array}{l}13 \\
38\end{array}$ & $\begin{array}{l}18 \\
55\end{array}$ \\
\hline
\end{tabular}

hospital response to hormone therapy has shown correlation with response to subsequent pituitary ablation by yttrium (Table III). Again Bulbrook et al. (1960) have established a correlation between the discriminant and the response to ablative operation. Thus a similar correlation seemed possible in relation to hormone therapy. This has not been established from our present findings.

\section{Summary}

The ratio of 11-deoxy-17-oxosteroids to 17-hydroxycorticosteroids (17-OHCS) has been recorded for urine specimens from 63 early and 107 late cases of breast cancer and from 37 normal women. In a proportion of these cases we have also arrived at values for the discriminant function $80-80(17-\mathrm{OHCS}$ mg. $/ 24 \mathrm{hr}$.) + aetiocholanolone $(\mu \mathrm{g} . / 24 \mathrm{hr}$.). A highly significant correlation between the ratio and the discriminant has been found.

We have attempted to investigate the value of the ratio as a prognostic index for cases of early breast cancer treated by surgery, radiotherapy, and $x$-ray menopause, and for late cases treated by $x$-ray menopause, hormone therapy, and chemotherapy. We have concluded that at the present time of assessment this approach has given us no useful prognostic indication in relation to such treatment. The evidence submitted does not permit appraisal of the correlation between the discriminant and response to endocrine ablative procedures reported by other workers.

We are grateful for the co-operation of Mr. R. Schofield, Department of Medical Illustration, and of Miss C. M. Evans, Department of Medical Statistics. Able technical assistance was given by Miss 
L. Procter and Miss S. Singer. Financial assistance from the Manchester Regional Hospital Board is acknowledged with thanks.

\section{REFERENCES}

Ahlquist, K. A. (1967). Proc. Ass. clin. Brochem., 4, 220.

Atkins, H. (1966). Ann. Roy. coll. Surg. Engl., 38, 133.

Bulbrook, R. D. (1965). Vitam. and Horm., 23, 329.

(1966). In Clinical Evaluation in Breast Cancer, edited by J. L. Hayward and R. D. Bulbrook, p. 78. London.

Greenwood, F. C. Band Hayward, J. L. (1960). Lancet, 1, 1154

and Hayward, J. L. (1966). In Androgens in Normal and Pathological Conditions, edited by A. Vermeulen and D. Exley, p. 101 Amsterdam.

- Spicer, C. C., and Thomas, B. S. (1962). Ibid., 2, 1238.

- and Thomas, B. S. (1964). Ibid., 1, 945.

Deshpande, N., Jenscn, V., Bulbrook, R. D., and Doouss, T. W. (1967). Steroids, 9,393 .
Few, J. D. (1961). 7. Endocr., 22, 31.

James, V. H. T., and De Jong, M. (1961). 7. clin. Path., 14, 425

Juret, P., Hayem, M., and Flaisler. A. (1964). J. Chir. (Paris), 87, 409 Kumaoka, S., Abe, A., Sakauchi, N., Takatani, O., and Kusama M (1966). Abstracts of 9th International Cancer Congress, Tokyo, (1966). Abstracts

Miller, H., Durant, J. A., Jacobs, A. G., and Allison, J. F. (1967). Brit. med. 7., 1, 147.

Paterson, R., and Russeli, M. H. (1959a). F. Fac. Radiol. (Lond.), 10, 175.

(1959b). Ibid., 10, 130.

Stempfel, R. S., iun., Sidbury, J. B., iun., and Migeon, C. J. (1960). f. clin. Endocr., 20, 814

Thomas, B. S., and Bulbrook, R. D. (1966). In Androgens in Normal and Pathological Conditions, edited by $A$. Vermeulen and D. Exley, p. 49. Amsterdam.

Wilson, R. E Hayward, J. L. (1967). Brit. med. 7., 3, 523. Emerson, K., and Moore, F., D. (1967), J., Bmer med Ass. 199., 474.

\title{
Rubella : a Method for Rapid Diagnosis of a Recent Infection by Demonstration of the IgM Antibodies
}

\author{
TIMO VESIKARI,* C.M.; ANTTI VAHERI, M.D.
}

Brit. med. F., 1968, 1, 221-223

Specific virological diagnosis of rubella is particularly important in pregnant women with a view to possible therapeutic abortion. This can be effectively done by using haemagglutinationinhibition (H.I.) or complement-fixation techniques, provided a serum pair taken in the acute and convalescent phases of the infection is available. However, as pointed out by Halonen et al. (1968), there always remain a certain number of rubella infections in which the first serum sample was taken too late to demonstrate any rise in antibody titre. Moreover, in pregnancies at the end of the third month even a week's delay ought to be avoided, because the therapeutic abortion should be applied for and executed as soon as possible. In these cases we should be able to distinguish a recent antibody response from previous experience with rubella. This cannot be concluded from the level of the H.I. titre alone, because there are individual variations in the response and because the titre seems to remain at a high value for a long time. Combination of the complementfixation method with crude complement-fixation antigen and the H.I. technique apparently does not provide any solution to this problem.

Earlier studies (Schluederberg, 1965) have demonstrated how the amount of $\operatorname{IgM}$ and $\operatorname{IgG}$ immune globulins varies in the course of viral infections. An initial production of $\operatorname{IgM}$ and subsequent IgG neutralizing rubella antibody was demonstrated previously in rubella-infected infants (Bellanti et al., 1965). The present paper reports the relative quantities of $\operatorname{IgM}$ and $\operatorname{IgG}$ H.I. antibodies in postnatal rubella infection, and describes a routine method for the diagnosis of rubella in which a single serum sample is used.

\section{Materials and Methods}

Treatment of Serum Samples.-Ordinarily $0.2 \mathrm{ml}$. of $1: 5$ dilution of untreated serum was used in the sedimentation analysis. In a few cases $0.1 \mathrm{ml}$. of undiluted serum was used in an attempt to demonstrate slight amounts of $\operatorname{IgM}$ antibody. To test the effect of kaolin some sera were treated as described in the rubella H.I. method of Stewart et al. (1967). To demon-

- University Department of Virology, and Virus Laboratory, Orion, Helsinki, Finland. strate sulphydryl reactivity treatment with $0.1 \mathrm{M}$ 2-mercaptoethanol for two hours at $37^{\circ} \mathrm{C}$. plus overnight dialysis against Dulbecco's phosphate buffered saline at $4^{\circ} \mathrm{C}$. was given. Both treatments resulted in a 1:5 dilution of the serum, and subsequently $0.2 \mathrm{ml}$. was analysed.

Sedimentation Analysis.-The samples were layered on top of linear $37-12.5 \% \mathrm{w} / \mathrm{v}$ sucrose gradients in phosphate-buffered saline (Vaerman et al., 1963) and centrifuged in a Spinco SW 39 rotor at 35,000 r.p.m. for $14 \frac{1}{2}$ hours. Twelve to 14 fractions were collected dropwise through the bottom of the tubes. The linearity of the gradients was checked by means of an Abbe refractometer.

Titration.-The original rubella H.I. method was employed (Stewart et al., 1967). The antigen was prepared in rubellainfected BHK 21/13 S suspension cultures (Vaheri et al., 1965) by treatment of the tissue culture fluid with Tween 80 and ether (Halonen et al., 1967). The H.I. titres varied between $1: 8$ and $1: 64$. The titrations were carried out on microtitre $\mathrm{V}$-plates with the use of a multimicrodiluter handle.

\section{Results}

Development of Antibody Response Pattern.-Five rubella cases in schoolchildren aged 10 were followed by serum samples taken at intervals after the onset of infection. (The total H.I. titres ranged at one week from 1:160 to $1: 640$, at three weeks from $1: 640$ to $1: 2,560$, and at six weeks from $1: 640$ to 1 : 2,560.) At one week (Fig. 1 A) a noticeable amount of IgM antibody was detected in all cases as well as IgG antibody. The relative quantity of IgG antibody was, however, always greater than that of IgM type. Three weeks after the rash the IgM antibody was still measurable in four out of five cases (Fig. 1 B). The fifth sample can apparently be regarded as an example of a weak IgM response, since such early disappearance of IgM immune globulin has not been found in any other rubella case studied so far. Six weeks after the rash there was no detectable amount of IgM in any serum (Fig. $1 \mathrm{C}$ ). In several other rubella cases with significant rise (fourfold) in total H.I. titre IgM antibody was constantly demonstrated in a serum sample obtained one to two weeks after the rash. 